\title{
AC/A RATIO
}

THE AC/A ratio is the relationship between the amount of convergence produced by the stimulus to accommodate (accommodative convergence, measured in prism dioptres) and the amount of accommodation (measured in dioptres) which produces that convergence.

Accommodation is the ability to adjust the curvature of the lens in order to focus rays of light on the retina, and convergence is the ability to maintain binocular fixation on an approaching object. Accommodation and convergence develop together so that a single clear image is appreciated. There are, however, other factors which affect convergence, and Maddox has described four stimuli to convergence:

(1) Accommodative convergence which is produced by the stimulus to accommodate.

(2) Tonic convergence occurs with consciousness, and the stimulus is from the neck muscles and labyrinths.

(3) Proximal convergence, in which the stimulus is the awareness of nearness.

(4) Fusional convergence, in which the stimulus is the desire for binocular single vision. Fusional convergence overcomes small degrees of heterophoria by the exertion of positive or negative relative convergence.

Convergence is generally measured in metre angles (M.A.), 1 metre angle being the amount of convergence which takes place between the two eyes when converging on an object 1 metre from the eyes (Fig. 1). The metre angle is not the same for every individual, being dependant on the interpupillary distance (IPD). The wider the IPD the greater the convergence necessary, and vice versa.

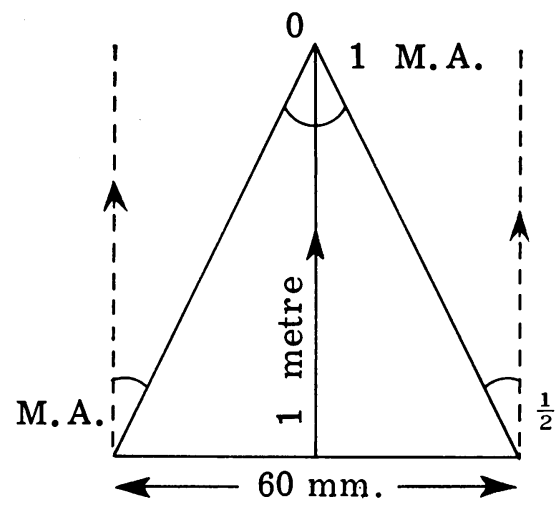

FIG. 1.-Measurement of convergence in metre angles.

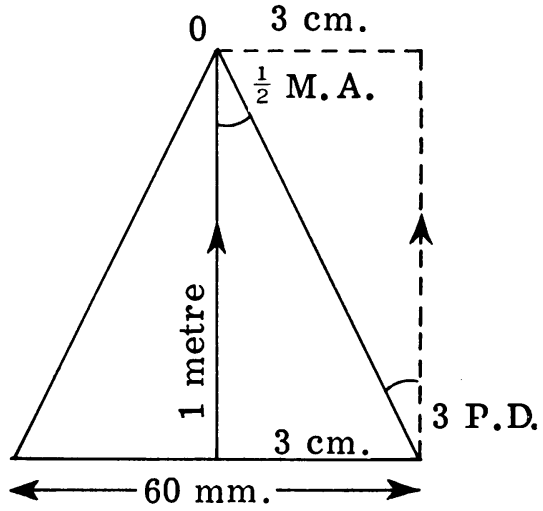

FIG. 2.-1 P.D. displaces an object at 1 metre by $1 \mathrm{~cm}$. 3 P.D. displaces an object at 1 metre by $3 \mathrm{~cm}$. 3 P.D. $=\frac{1}{2}$ metre angle (as shown) 6 P.D. $=1$ metre angle

Accommodation and convergence are inter-related so that a single clear image can be appreciated. To see something clearly and singly at 1 metre, 1 dioptre of accommodation and 1 metre angle of convergence are exerted. This is known as the $1: 1$ relationship. Clinically it is easier to compare one person with another by measuring convergence in prism dioptres (P.D.). 6 prism dioptres $=1$ metre angle when the IPD is $60 \mathrm{~mm}$. (see Fig. 2). To see clearly and singly at 1 metre, 6 prism dioptres of convergence are therefore exerted with 1 dioptre of accommodation, this 6 prism dioptres being the total amount of convergence. One-third of convergence that takes place ( 2 prism dioptres) is tonic, proximal, and fusional, while two-thirds ( 4 prism dioptres) are accommodative.

4 prism dioptres of accommodative convergence: 1 dioptre of accommodation, therefore the $\mathrm{AC} / \mathrm{A}$ ratio $=4: 1$.

This is a theoretical concept and the AC/A ratio is considered normal between $3: 1$ and $5: 1$.

\section{Measurement of the AC/A Ratio}

The aim is to obtain the relationship between accommodative convergence and accommodation when proximal and fusional convergence are eliminated. Proximal convergence is eliminated by 
using a distant object and fusional convergence by dissociating the eyes. There are numerical and graphic methods for measuring the $\mathrm{AC} / \mathrm{A}$ ratio, and the following are the more common ones used:

(1) Numerical

(a) Gradient Method with Prisms.-The deviation at 6 metres is measured with prisms, using the smallest Snellen's type possible. Measurements are made firstly with glasses if worn, and then with minus spheres (e.g. $-3.0 \mathrm{D}$ sph.).

$$
\mathrm{AC} / \mathrm{A}=\frac{\Delta \mathbf{D}-\Delta}{\mathbf{D}} \quad \begin{aligned}
\Delta & =\text { deviation at } 6 \text { metres } \\
\Delta \mathbf{D} & =\text { deviation at } 6 \mathrm{~m} . \text { with }-3 \mathrm{D} \text { sph. } \\
\mathbf{D} & =\text { strength of minus lenses used. }
\end{aligned}
$$

Although this method eliminates proximal and fusional convergence it is not as accurate as the graphic method as it is not possible to determine whether the relationship is linear.

(b) Heterophoria Method (Burian).-The deviation is measured at 6 metres with $6 / 6$ Snellen's test type and then at $\frac{1}{3}$ metre with $6 / 6$ reduced Snellen's type.

$$
\mathrm{AC} / \mathrm{A}=1 \text { P.D. }+\frac{(\Delta \mathrm{n}-\Delta)}{\mathrm{N}} \quad \begin{aligned}
\Delta \mathrm{n} & =\text { deviation for near } \\
\Delta & =\text { deviation for distance } \\
\mathbf{N} & =\text { accommodation exerted (i.e. } 3 \text { dioptres at } \frac{1}{3} \text { metre) }
\end{aligned}
$$

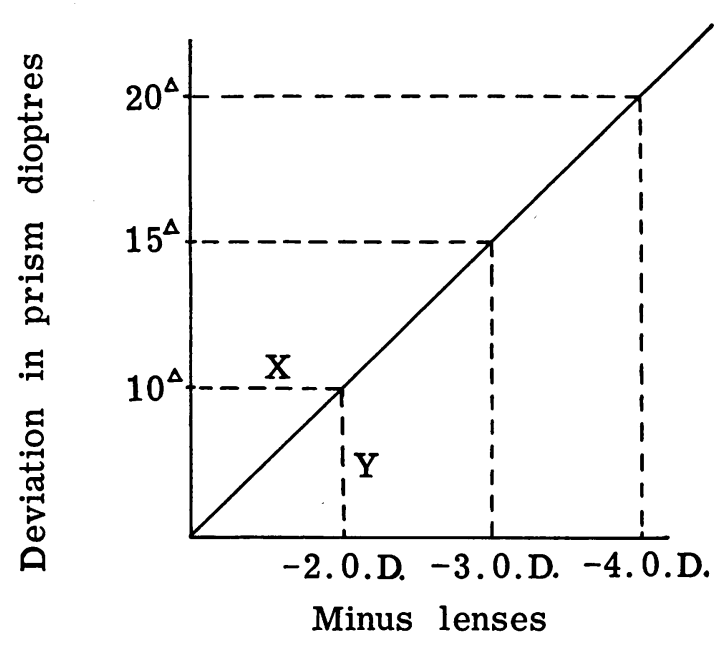

FIG. 3. $-\mathrm{AC} / \mathrm{A}=\mathrm{X} / \mathrm{Y}$

(2) Graphic

The deviation at 6 metres is measured with prisms, using the smallest Snellen's type possible. Measurements are made firstly with glasses if worn and then with increasing strengths of minus lenses. The $\mathrm{AC} / \mathrm{A}$ ratio is then calculated from the graph $\mathrm{AC} / \mathrm{A}=\mathrm{X} / \mathrm{Y}$ (see Fig. 3).

\section{Significance of the AC/A Ratio}

If the AC/A ratio is abnormally high, excessive accommodative convergence is exerted on accommodation. A high AC/A ratio is found in patients with accommodative squints of the convergence excess type. If the AC/A ratio is low, less accommodative convergence takes place on accommodation and the eyes will be relatively divergent. Although the $\mathrm{AC} / \mathrm{A}$ ratio is an important factor in determining the type of deviation present, its significance in both the aetiology and treatment of squints is debatable. It is believed that the AC/A ratio is inborn and remains constant throughout life.

\section{Effect on AC/A Ratio of Miotic Drugs}

Miotic drugs put the ciliary muscle into spasm so that some of the accommodation necessary for clear vision for near is exerted passively. Only active accommodation results in accommodative convergence as the AC/A ratio is a central relationship. If the miotic exerts $\frac{1}{2}$ dioptre of accommodation and the AC/A ratio is $10: 1$, the $\frac{1}{2}$ dioptre of accommodation results in 5 prism dioptres of convergence and the patient should be able to control the reduced deviation when accommodation is exerted. This control often remains when the miotic is gradually discontinued. In many cases, however, the miotic seems merely to mask the high AC/A ratio so that when it is discontinued the deviation returns.

Illustrations:

INSTITUTE OF OPHTHALMOLOGY, JudD ST., LONDON, W.C.1.
ANN Hughes,

ORTHOPTIC DepartMENT, Moorfields Eye Hospital, LONDON, W.C.1. 